\title{
Output of an Ultrasonic Wave-Driven Nanogenerator in a Confined Tube
}

\author{
Xudong Wang ${ }^{1,2}$, Yifan Gao ${ }^{1}$, Yaguang Wei', and Zhong Lin Wang ${ }^{1}(\varangle)$ \\ ${ }^{1}$ School of Materials Science and Engineering, Georgia Institute of Technology, Atlanta, Georgia 30332-0245, USA \\ ${ }^{2}$ Department of Materials Science and Engineering, University of Wisconsin at Madison, Madison, Wisconsin 53706, USA \\ Received: 5 December 2008/Revised: 8 January 2009/Accepted: 9 January 2009 \\ CTsinghua University Press and Springer-Verlag 2009. This article is published with open access at Springerlink.com
}

\begin{abstract}
The output of an ultrasonic wave-driven nanogenerator (NG) has been found to depend on the excitation conditions and geometry. Incidence angle tests indicate that the effective area of an NG determines the amount of power that can be generated. The output power of an NG is also directly related to its distance from the ultrasonic source. A sinusoidal profile of the electrical output was observed when an NG was moved inside a long tube filled with water with the ultrasonic source located at one end. This is due to the oscillation of the wave intensity inside the tube as a function of the distance from the excitation source.
\end{abstract}

\section{KEYWORDS}

Nanogenerator, ultrasonic wave, $\mathrm{ZnO}$, nanowire

Harvesting energy from the environment is critical for the sustainable operation of wireless sensor systems and implantable biomedical devices [1-3]. Mechanical energy is the most ubiquitous energy source in our living environment that can be readily collected and converted into electricity [4]. A piezoelectric cantilever-based micro electromechanical system (MEMS) is, for example, one of the most common techniques for mechanical energy harvesting [5]. Flexible piezoelectric thin films are also increasingly used for practical applications [6, 7]. Vibrating a metal coil in a magnetic field has been demonstrated to give mechanical energy conversion through electromagnetic induction [8]. These approaches have been developed for micro-scale devices for which gravitation or inertia can play a significant role. As for nano-scale devices and structures, previously demonstrated methodologies and principles may not be applicable due to the change in operation frequencies and elimination of the role played by gravitation. A recent development of a nanogenerator (NG) based on nanowires (NWs) has shed light on the harvesting of mechanical energy using nanotechnology [9]. This technique relies on coupling of the piezoelectric and semiconductor properties of ZnO NWs [2, 10], which allows a scavenging of mechanical energy at the nanometer scale in the form of waves [11] or pulses [12] with high and low frequencies. One of the most important advantages of the NG is that its operation does not rely on resonance but on mechanical disturbance or vibration over a large frequency range.

Driving an NG indirectly through a sonic/ ultrasonic wave is ideal for remote powering or even

Address correspondence to zlwang@gatech.edu 
self-sufficient operation of a nanosystem. Applying ultrasonic waves (USWs) through liquid media has been demonstrated to be an effective way for agitating NGs [13]. Understanding the relationship between a USW and the output of an NG is of significant importance if the output power of the NG is to be increased to meet the needs of a self-powered nanosystem. In this paper, we describe a study of the interaction of USWs with an NG and its dependence on the wave incidence angle and the distance from the wave source. A comparison of the experimental data with theoretical simulations directly proves the relationship between the input wave energy and the output power.

The NGs used in our experiments were all assembled on glass substrates by the technique reported previously [11]. The experiment setup for the incidence angle tests is shown schematically in the inset on the left hand side of Fig. 1(a). The NG sample was attached to the glass sample holder, which can be rotated freely. The incidence angle test was performed in a large water cavity, where a USW source of frequency $\sim 40 \mathrm{kHz}$ was located at the bottom center. The center of the NG was fixed at the center of the cavity and was kept at $2 \mathrm{~cm}$ below the water surface. The size of the NG was $2.5 \mathrm{~mm}$ $\times 2.5 \mathrm{~mm}$, which is much smaller than the size of the water tank. The incidence angle is defined as the angle between the glass sample holder and the horizontal position when the NG is rotated laterally around its horizontal axis. It should be noted that both sides of the NG are not identical, as shown in the right hand side of Fig. 1(a). The active part of the NG is the zigzag electrode; this is covered by epoxy, which seals the edge to prevent water infiltration and is defined as the top side. The bottom side of the NG is the glass substrate. Therefore, an incidence angle of $0^{\circ}$ corresponds to the case that the top side of the NG is facing the water surface, while an angle of $180^{\circ}$ indicates that the top side is facing the wave source.

Five NG samples were tested individually at nine incidence angles of $0^{\circ}, 30^{\circ}, 45^{\circ}, 60^{\circ}, 90^{\circ}, 120^{\circ}, 135^{\circ}$, $150^{\circ}$, and $180^{\circ}$. The short circuit current $\left(I_{\mathrm{SC}}\right)$ was recorded to show the output change when the USW source was turned on and off. A typical set of angular dependent $I_{\mathrm{SC}}$ data is shown in Fig. 1(b). As the

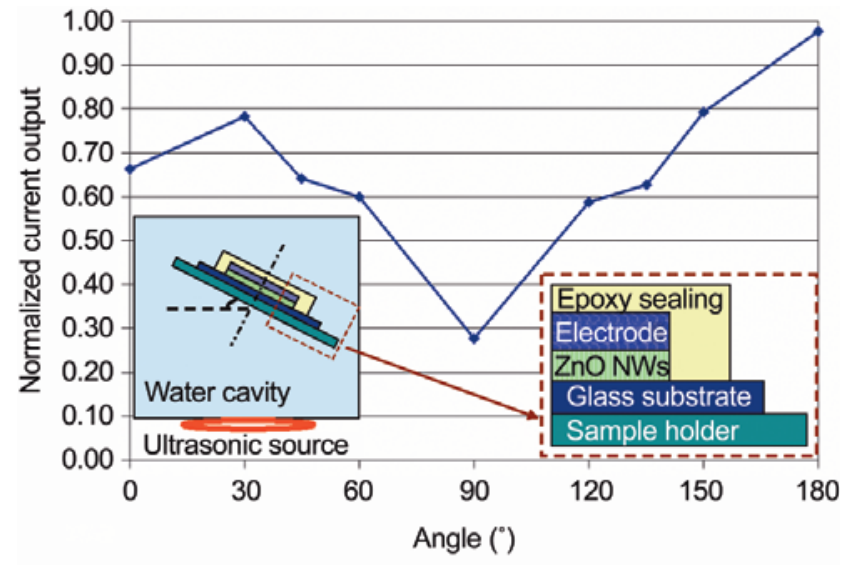

(a)

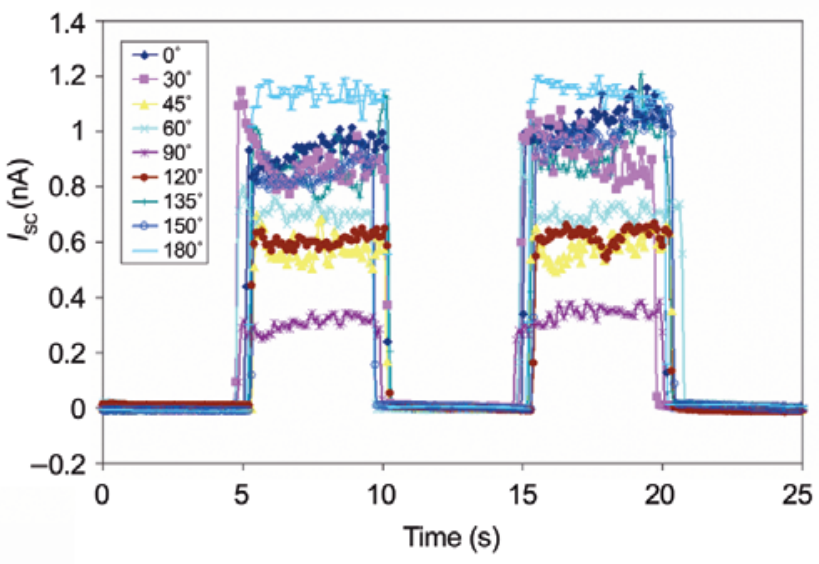

(b)

Figure 1 (a) Plot of the normalized $I_{s c}$ and $V_{\text {oc }}$ signals of five NGs at different incidence angles. The schematic experimental setup is shown in the inset on the left hand side. The structure of the NG is shown on the right hand side. (b) One set of $I_{\text {sc }}$ output data for an NG measured at nine different incidence angles

USW was turned on, the current signal immediately jumped to a higher level and remained steady until the USW was turned off. Different incidence angles resulted in distinct $I_{\mathrm{SC}}$ values and the lowest and highest outputs were given by $90^{\circ}$ and $180^{\circ}$, respectively. Since the output signals are rather noisy, the $I_{\mathrm{SC}}$ and $V_{\mathrm{OC}}$ values were treated by removing the averaged base line from the averaged signal amplitude. The complete set of angular dependent $I_{\mathrm{SC}}$ data measured from the five samples is listed in Table 1. The averaged $I_{\mathrm{SC}}$ value varied from tens of $\mathrm{pA}$ to one $\mathrm{nA}$, and the output difference between each sample is attributed to the quality and uniformity of NWs, contact resistance, and the assembly and packaging processes. The details of these effects can be found elsewhere [14]. 
Table 1 Dependence of averaged short circuit current output (in $\mathrm{nA}$ ) on wave incidence angle

\begin{tabular}{cccccc}
\hline$\alpha\left(^{\circ}\right)$ & \multicolumn{3}{c}{$I_{\text {sc }}(\mathrm{nA})$} \\
\cline { 2 - 6 } & Sample \#1 & Sample \#2 & Sample \#3 & Sample \#4 & Sample \#5 \\
\hline 0 & $0.98 \pm 0.07$ & $0.014 \pm 0.001$ & $0.049 \pm 0.006$ & $0.044 \pm 0.0008$ & $0.020 \pm 0.004$ \\
30 & $0.90 \pm 0.08$ & $0.017 \pm 0.003$ & $0.064 \pm 0.01$ & $0.055 \pm 0.002$ & $0.027 \pm 0.006$ \\
45 & $0.58 \pm 0.05$ & $0.019 \pm 0.001$ & $0.039 \pm 0.004$ & $0.042 \pm 0.002$ & $0.023 \pm 0.004$ \\
60 & $0.71 \pm 0.04$ & $0.015 \pm 0.002$ & $0.020 \pm 0.005$ & $0.038 \pm 0.002$ & $0.025 \pm 0.005$ \\
90 & $0.32 \pm 0.03$ & $0.012 \pm 0.001$ & $0.015 \pm 0.003$ & $0.005 \pm 0.0006$ & $0.008 \pm 0.001$ \\
120 & $0.61 \pm 0.03$ & $0.018 \pm 0.002$ & $0.030 \pm 0.005$ & $0.038 \pm 0.0007$ & $0.019 \pm 0.002$ \\
135 & $0.91 \pm 0.09$ & $0.014 \pm 0.002$ & $0.039 \pm 0.007$ & $0.039 \pm 0.0008$ & $0.021 \pm 0.004$ \\
150 & $0.93 \pm 0.09$ & $0.013 \pm 0.002$ & $0.094 \pm 0.02$ & $0.051 \pm 0.0007$ & $0.028 \pm 0.003$ \\
180 & $1.14 \pm 0.04$ & $0.016 \pm 0.001$ & $0.13 \pm 0.009$ & $0.071 \pm 0.002$ & $0.029 \pm 0.004$ \\
\hline
\end{tabular}

Although the individual output values exhibited some variation, they showed similar patterns at different incidence angles. The general relationship between the incidence angle and the output can be revealed by normalizing with respect to the maximum output at $180^{\circ}$, as shown in Fig. 1(a). The lowest output is found at an incidence angle of $90^{\circ}$, which is when the NG is placed perpendicular to the water surface. When the NG was rotated clockwise from $90^{\circ}$ to $30^{\circ}$ or rotated counter-clockwise from $90^{\circ}$ to $150^{\circ}$, the output showed a symmetric increase. The output reached a maximum at $180^{\circ}$, at which position the NG had its zigzag electrode directly facing the incoming USW. However, a slight drop was found when the NG had its glass substrate side facing the incoming USW $\left(0^{\circ}\right)$.

The orientation-related output change can be attributed to the effective surface area of an NG available to receive the USW. When a wave impinges on an NG, energy is transferred from wave to NG. Suppose the area of the NG is $A$, and the wave propagation $k$ direction is at an angle of $\alpha$ to the normal of this area. The effective cross section area which absorbs energy is $A_{\text {eff }}=A|\cos \alpha|$. Therefore, the output is maximized when $\alpha=0^{\circ}$ or $180^{\circ}$ and minimized when $\alpha=90^{\circ}$, since

$$
P_{\text {out }}(\alpha)=\mathrm{P}(0) \cdot|\cos \alpha|
$$

Equation (1) applies to an ideal case for straight propagation of the USW with no side propagation or reflection taking place. Thus, when $\alpha=90^{\circ}$, there is no interaction between the USW and the NG, which should result in zero output. However, in a real case, even when the NG was positioned perpendicular to the water surface, a significant output was still recorded. This is due to the existence of some nonperpendicular propagated waves in the water cavity. The effect of such waves only becomes significant when the interaction from the perpendicularly propagated wave becomes small, i.e., when $\alpha$ is close to $90^{\circ}$. The decrease in signal at $0^{\circ}$ is probably due to the damping effect from the double glass substrate, which absorbs part of the ultrasonic energy before it reachs the NG core.

After revealing the role of the effective area in NG operation, we further investigated the importance of effective distance for remote powering of an NG. The experimental setup is shown in Fig. 2(a). In an ultrasonic generator chamber filled with water, a 30 $\mathrm{cm}$ long glass tube was vertically placed at the point where the ultrasonic source is generated. The glass tube was also filled with water. An NG was placed inside the tube at a fixed angle of $45^{\circ}$ from the vertical direction. The $I_{\mathrm{SC}}$ and / or $V_{\mathrm{OC}}$ were continuously monitored while the NG was moved freely straight up and down in the tube with a constant speed and unaltered lateral position. The operation distance, $d$, was defined as the distance from the center of the NG to the bottom of the tube.

One typical output pattern is shown in Fig. 2(b). The NG was initially located at the bottom of the tube. When the USW was turned on, the $I_{\mathrm{SC}}$ signal immediately jumped to, and then stayed 


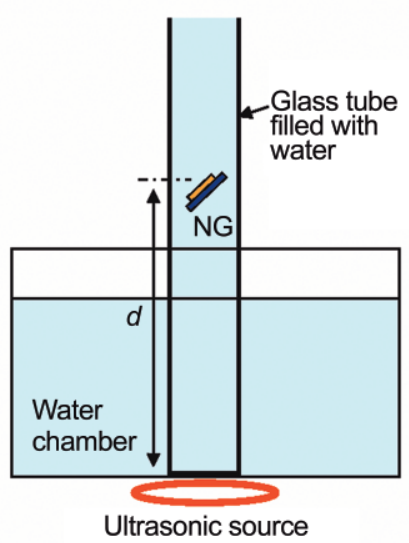

(a)

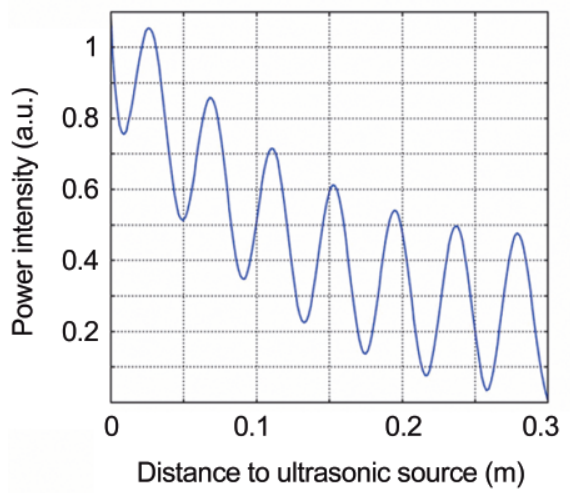

(d)

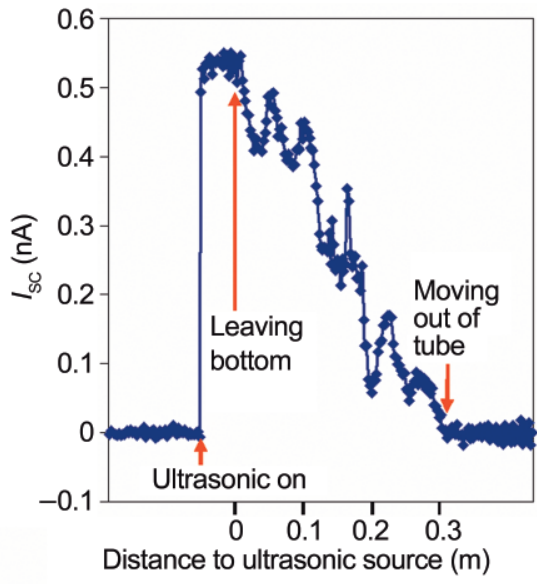

(b)

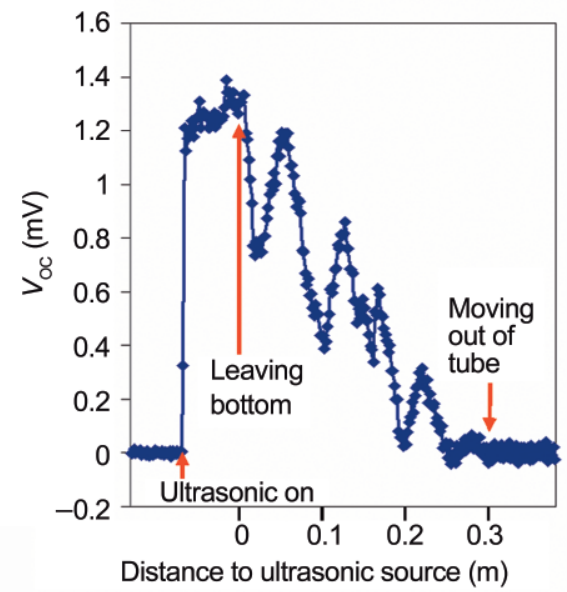

(c)

Figure 2 (a) Schematic experimental setup for measuring the output dependence of an NG on the distance from the source of the USW; typical (b) $I_{S c}$ and (c) $V_{o c}$ output patterns recorded when an NG was moved from bottom to top in the tube of water; (d) the simulated USW intensity profile along the axis of a $30 \mathrm{~cm}$ long tube obtained by integrating the intensity over the cross section of the NG

at its maximum. As the NG moved away from the ultrasonic source, the intensity decreased and oscillated in a sinusoidal-like pattern and finally reached the baseline when the NG was moved out of the tube. The same pattern was observed for the $V_{\mathrm{OC}}$ signal (Fig. 2(c)). In a $30 \mathrm{~cm}$ long path, typically 5 or 6 peaks can be identified. The size of the NG was 25 $\mathrm{mm} \times 25 \mathrm{~mm}$.

The oscillation of the output voltage as a function of the distance from the center of the USW is suggested to be due to the change in stationary wave intensity inside the tube as confined by the boundary of the tube. For a tubular shaped structure, stationary acoustic waves are excited inside the tube by the USW source. The USW intensity profile in the testing tube follows a sinusoidal function with its amplitude decaying as a function of distance from the USW source. This is what we have observed in Fig. 2. Theoretical modeling revealed the sinusoidal profile of the distance-dependent output. The USW intensity profile in the testing tube can be simulated with a simple acoustic model. The ultrasonic source used in our experiments was $f=\frac{\omega}{2 \pi}=40 \mathrm{kHz}$, the sound pressureis $p$ in the form of $p(r, t)=p(r) \mathrm{e}^{\mathrm{i} w t}$, and the amplitude is determined by the Helmholtz equation:

$$
\nabla^{2} p+\frac{\omega^{2}}{c^{2}} p=0
$$

where $c=1497 \mathrm{~m} / \mathrm{s}$ is the speed of sound in water. We assume that the USW impinges into the tube 
from the bottom. The top, therefore, is far from the USW source and satisfies the soft acoustic boundary condition. The boundary condition on the side wall of the tube can thus be modeled as a finite impedance boundary. For a tube with diameter $d=3.2 \mathrm{~cm}$ diameter and length $l=30 \mathrm{~cm}$, the distribution of the intensity oscillates as a function of distance from the USW source and decays (Fig. 2(d)). A more careful inspection shows that there are 7 maxima in Fig. 2(d), whereas we only observed 5 or 6 peaks for most of the NGs (Figs. 2(b) and 2(c)). This difference may be due to changes in the resonance mode in the tube, caused by the introduction of the NG together with the glass substrate, that alter the number of stationary peaks.

The oscillating output voltage/current was further confirmed by using a $70 \mathrm{~cm}$ long tube, where the size effect of the NG can be ignored. An NG was tested inside this tube while it was moved away or towards the ultrasonic source. The corresponding $I_{\mathrm{SC}}$ signals are shown in Figs. 3(a) and 3(b), respectively. The current profiles exhibit a very similar pattern to the simulations, irrespective of the direction of motion of the NG. Furthermore, 17 peaks can be observed in both $I_{\mathrm{SC}}$ profiles, which scales approximately in reference to the length of the tube. With its larger volume, the tube provides a more stable path for the USW to propagate. Even when an NG was placed inside, its size effect was negligible relative to the large size of the tube. The sinusoidal output pattern proved that the output of the NG is directly related to the intensity of the USW. The more energy supplied by the ultrasonic source, the greater the induced displacement of the NWs in the NG. In the sandwich structure of NWs, most of the rigidity comes from the packaging material. Our results therefore suggest that more flexible packaging and spacing materials could possibly improve the NG output efficiency by a significant amount.

In summary, the output of NGs driven by USWs has been studied systematically. The incidence angle tests revealed that the effective area of an NG determines the amount of power that can be generated. The output energy of an NG is also directly related to the distance from the ultrasonic source. A sinusoidal profile of the electrical output

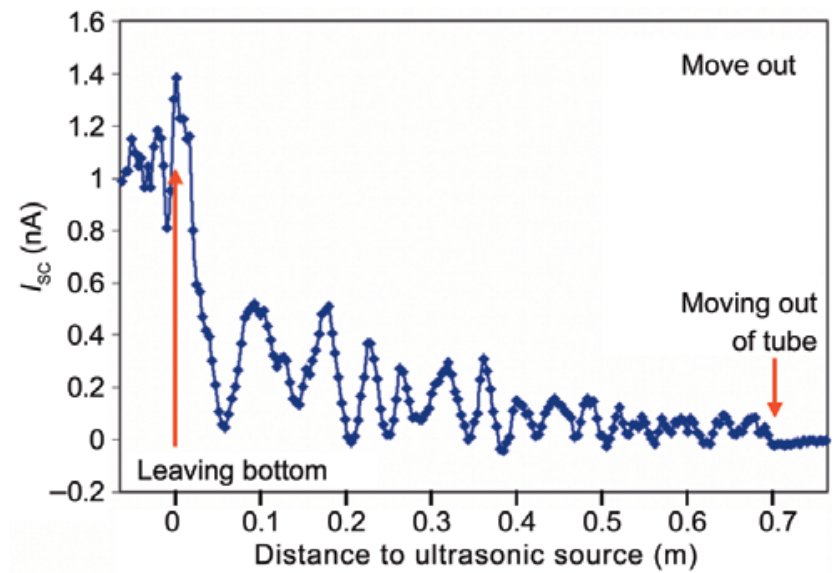

(a)

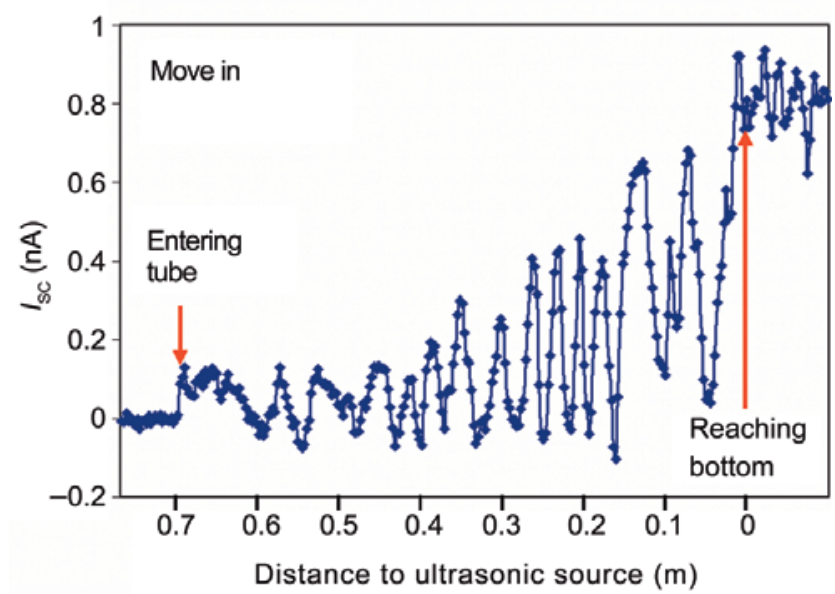

(b)

Figure 3 Typical $I_{s c}$ output patterns recorded when an NG was (a) pulled out from bottom to top and (b) moved in from top to bottom in a $70 \mathrm{~cm}$ long tube

was observed when an NG was moved inside a long tube with the ultrasonic source located at one end. The oscillation of the output voltage/current is related to the intensity of the local USW. The discovery of the angular and distance dependent output of NGs provides valuable guidance for future NG characterization and operation. In order to test or operate an NG at its highest capability, the NG should have its surface normal to the incident USW and be located at the position where the USW exhibits the strongest intensity.

\section{Acknowledgements}

This research was sponsored by BES DOE and Medtronic, Inc. 


\section{References}

[1] Wang, Z. L. Self-powered nanotech. Sci. Am. 2008, 298, 82-87.

[2] Wang, Z. L. Towards self-powered nanosystems: From nanogenerators to nanopiezotronics. Adv. Funct. Mater. 2008, 18, 3553-3567.

[3] Tian, B.; Zheng, X.; Kempa, T. J.; Fang, Y.; Yu, N.; Yu, G.; Huang, J.; Lieber, C. M. Coaxial silicon nanowires as solar cells and nanoelectronic power sources. Nature 2007, 449, 885-890.

[4] Paradiso, J. A.; Starner, T. Energy scavenging for mobile and wireless electronics. IEEE Pervas. Comput. 2005, 14, 18-27.

[5] Roundy, S.; Wright, P. K. A piezoelectric vibration-based generator for wireless electronics. Smart Mater. Struct. 2004, 13, 1131-1142.

[6] Donelan, J. M.; Li, Q.; Naing, V.; Hoffer, J. A.; Weber, D. J.; Kuo, A. D. Biomechanical energy harvesting: Generating electricity during walking with minimal user effort. Science 2008, 319, 807-810.

[7] Granstrom, J.; Feenstra, J.; Sodano, H. A.; Farinholt, K. Energy harvesting from a backpack instrumented with piezoelectric shoulder straps. Smart Mater. Struct. 2007,
16, 1810-1820.

[8] Williams, C. B.; Yates, R. B. Analysis of a micro-electric generator for microsystems. Sensor. Actuat. A-Phys. 1996, 52, 8-11.

[9] Wang, Z. L.; Song, J. H. Piezoelectric nanogenerators based on zinc oxide nanowire arrays. Science 2006, 312, 242-246.

[10] Song, J. H.; Zhou, J.; Wang, Z. L. Piezoelectric and semiconducting coupled power generating process of a single $\mathrm{ZnO}$ belt/wire: A technology for harvesting electricity from the environment. Nano Lett. 2006, 6, 1656-1662.

[11] Wang, X. D.; Song, J. H.; Liu, J.; Wang, Z. L. Directcurrent nanogenerator driven by ultrasonic waves. Science 2007, 316, 102-105.

[12] Qin, Y., Wang, X. D.; Wang, Z. L. Microfiber-nanowire hybrid structure for energy scavenging. Nature 2008, 451, 809-813.

[13] Wang, X. D.; Liu, J.; Song, J. H; Wang, Z. L. Integrated nanogenerators in biofluid. Nano Lett. 2007, 7, 24752479.

[14] Liu, J.; Fei, P.; Zhou, J.; Tummala, R.; Wang, Z. L. Toward high output-power nanogenerator. Appl. Phys. Lett. 2008, 92, 173105 . 